\title{
The CDF II eXtremely Fast Tracker Upgrade
}

A. Abulencia ${ }^{\mathrm{a}}$, P. Azzurri ${ }^{\mathrm{b}}$, E. Cochran ${ }^{\mathrm{c}}$, J. Dittmann ${ }^{\mathrm{d}}$, S. Donati ${ }^{\mathrm{b}}$, J. Efron ${ }^{\mathrm{c}}$, R. Erbacher ${ }^{\mathrm{e}}$, D. Errede $^{\mathrm{a}}$, I. Fedorko ${ }^{*}$, G. Flanagan ${ }^{\mathrm{g}}$, R. Forrest ${ }^{\mathrm{e}}$, J. Gartner ${ }^{\mathrm{c}}$, H. Gerberich ${ }^{\mathrm{a}}$, N. Giokaris ${ }^{\mathrm{f}}, \mathrm{S}$.

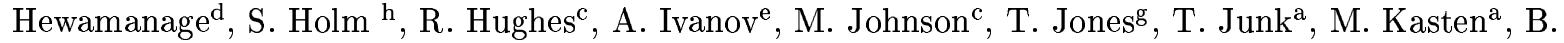
Kilminster $^{\mathrm{c}}$, R. Klein ${ }^{\mathrm{h}}$, N. Krumnack ${ }^{\mathrm{d}}$, K. Lannon ${ }^{\mathrm{c}}$, S. Levine ${ }^{\mathrm{a}}$, J. McKimc ${ }^{\mathrm{c}}$, R. Mokos ${ }^{\mathrm{a}}$, D. Olivito ${ }^{\mathrm{c}}$, B.

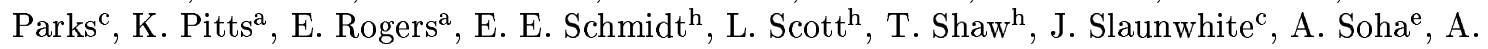

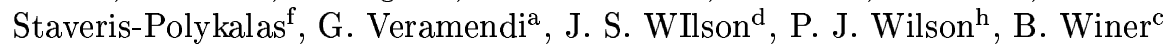

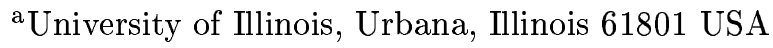

${ }^{\mathrm{b}}$ Istituto Nazionale di Fisica Nucleare Pisa, Universities of Pisa, Siena and Scuola Normale Superiore, I-56127 Pisa, Italy

${ }^{\mathrm{c}}$ The Ohio State University, Columbus, Ohio 43210

${ }^{\mathrm{d}}$ Baylor University, Waco, Texas 76796

eUniversity of California, Davis, Davis, California 95616

${ }^{\mathrm{f}}$ National and Capodistrian Univerity of Athens, Athens 10679, Greece, and Istituto Nazionale di Fisica Nucleare, Pisa, I-56127, Italy

gPurdue University, West Lafayette, Indiana 47907

${ }^{\mathrm{h}}$ Fermi National Accelerator Laboratory, Batavia, Illinois 60510,USA

The CDF II eXtremely Fast Tracker is the trigger track processor which reconstructs charged particle tracks in the transverse plane of the CDF II central outer tracking chamber. The system is now being upgraded to perform a three dimensional track reconstruction. A review of the upgrade is presented here.

\section{Introduction}

The eXtremely Fast Tracker [1] finds tracks in the transverse plane of the CDF II Central Outer Chamber (COT) [2] in time for the Level 1 trigger decision. The XFT tracks are also matched to electromagnetic-calorimeter clusters for online electron identification, and to stubs in the muon system for muon identification. The role of the $\mathrm{XFT}$ in the trigger is to provide useful information to reduce the raw collision rate $(1.7 \mathrm{MHz})$ to a maximum of about $30 \mathrm{KHz}$ of more "interesting" physics events which can be processed by the Level 2 trigger. The XFT currently uses hit data

${ }^{*}$ Corresponding author.

email address: ivan.fedorko@pi.infn.it from the 4 axial superlayers of the COT, which are arranged in cells of 12 wires each, for a total of 16,128 axial wires. A charged track passing through an axial layer generates a characteristic pattern of 12 hits, one per wire, with a characteristic timing. Track identification is performed in two processes by the Finder, which searches for track segments in each of the 4 axial layers of the chamber, and by the Linker, which searches for $4 / 4$ as well as $3 / 4$ matches among segments in the 4 layers, consistent with a track exiting from the interaction point. The XFT measures the transverse momentum and azimuthal angle of all the tracks with $p_{T}>1.5 \mathrm{GeV} / \mathrm{c}$ with an efficiency greater than $96 \%$ per track, and resolution $\sigma_{p_{T}} / p_{T}^{2} \sim 2 \%\left(\mathrm{GeV}^{-1}\right)$ and $\sigma_{\phi} \sim 6 \mathrm{mR}$. 


\subsection{System architecture}

The XFT system architecture is presented schematically in Fig. 1. The XTC mezzanine cards reside on the COT TDC's and classify the hits on each COT wire as prompt or delayed depending on whether the time of the hit is smaller or larger than $44 \mathrm{~ns}$, respectively. These data are sent to a transition module on the back of the COT TDC crate and are sent at $45.5 \mathrm{MHz}$ as LVDS signals to the Finder module crates, where they are received by transition modules and go to the Finder modules through a customized backplane. The Finder modules find track segments and transmit them to the Linker modules which find the tracks and the associated parameters $p_{T}$ and $\phi$. Track information is driven by transition modules to the extrapolation unit (XTRP) which extrapolates the tracks to the electromagnetic calorimeter and the muon chambers and distributes the data to the Level 1 and Level 2 triggers. At Level 2 the XFT tracks are used as seeds by the Online Silicon Vertex Tracker [3] which performs a combined track reconstruction in the tracking chamber and in the silicon vertex detector.

\section{The upgrade of the system}

The XFT was designed to perform online tracking at an instantaneous luminosity of about $10^{32}$ $\mathrm{cm}^{-2} \mathrm{~s}^{-1}$. The increase above this limit demands an upgrade of the system to cope with the increased occupancy of the chamber, which results in a higher level of fake tracks and consequently in larger rates of the triggers based on the XFT tracks. The upgrade foresees that the existing axial system is not altered, and new Stereo Finder boards are added to find track segments in the three outer stereo layers of the COT. The idea is to reject fake axial tracks by requiring the association of the axial tracks with the stereo segments. The stereo upgrade also allows the measurement of the track parameters $\cot \theta$ and $z_{0}$, which can be used to require the XFT track to point in the $z$-direction towards the calorimeter tower or the muon stub involved in electron or muon triggers.

\subsection{Upgraded system architecture}

The upgraded system architecture is presented schematically in Fig. 1, where the shaded boxes indicate the new components. The XTC-2 mezzanine cards reside on the TDC's that process data from the COT stereo layers. The card classifies the hits in 6 time windows with programmable edges, set around 55, 79, 103, 127, 151 and 255 ns. Data are transmitted through additional mezzanine cards installed at the back of the TDC crates to the Stereo Finders using optical fibers. The Stereo Finder boards identify track segments in the stereo layers of the COT. Each board covers a $30^{\circ}$ azimuthal angle slice, so a total of 36 boards is necessary to instrument the 3 outer stereo layers. The number of 12 wire cells processed by each board is respectively $20 / 28 / 36$ for the internal/central/external stereo layers. The Stereo Finder logic is implemented with two Altera Stratix EP2S60 Programmable Gate Arrays. The Stereo Finders output the list of found pixels, which indicate the position and slope of an identified track segment. The pixels exist in two formats output through two independent data streams on optical fibers. The first one has each COT cell divided in 12 pixels, with 6 azimuthal bins and 2 slope bins, and is directed to the Stereo Linker Association Modules (SLAM), which perform the matching between the axial XFT tracks and the stereo pixels. The second one, less compressed, has each COT cell divided in 90 pixels, with 18 azimuthal bins and 5 slope bins, and is directed to the Level 2 trigger processors, where the extra time available allows the use of more refined information. Each SLAM receives the axial track data from one Linker Module, which covers a $15^{\circ}$ azimuthal angle slice, and the stereo pixels from a slice of $45^{\circ}$ azimuthal angle covered by two Stereo Finders and centered on the region covered by the Linker Module. The association algorithm is implemented using one $\mathrm{Al}$ tera Stratix EP1S25 per module. The format of the data output from the SLAM to the extrapolation unit (XTRP) is identical to the original output of the Linker Output Modules, with the insertion of the "matched/unmatched track" information and the "positive/negative $z$ direction" in two presently spare bits. At high luminosity 


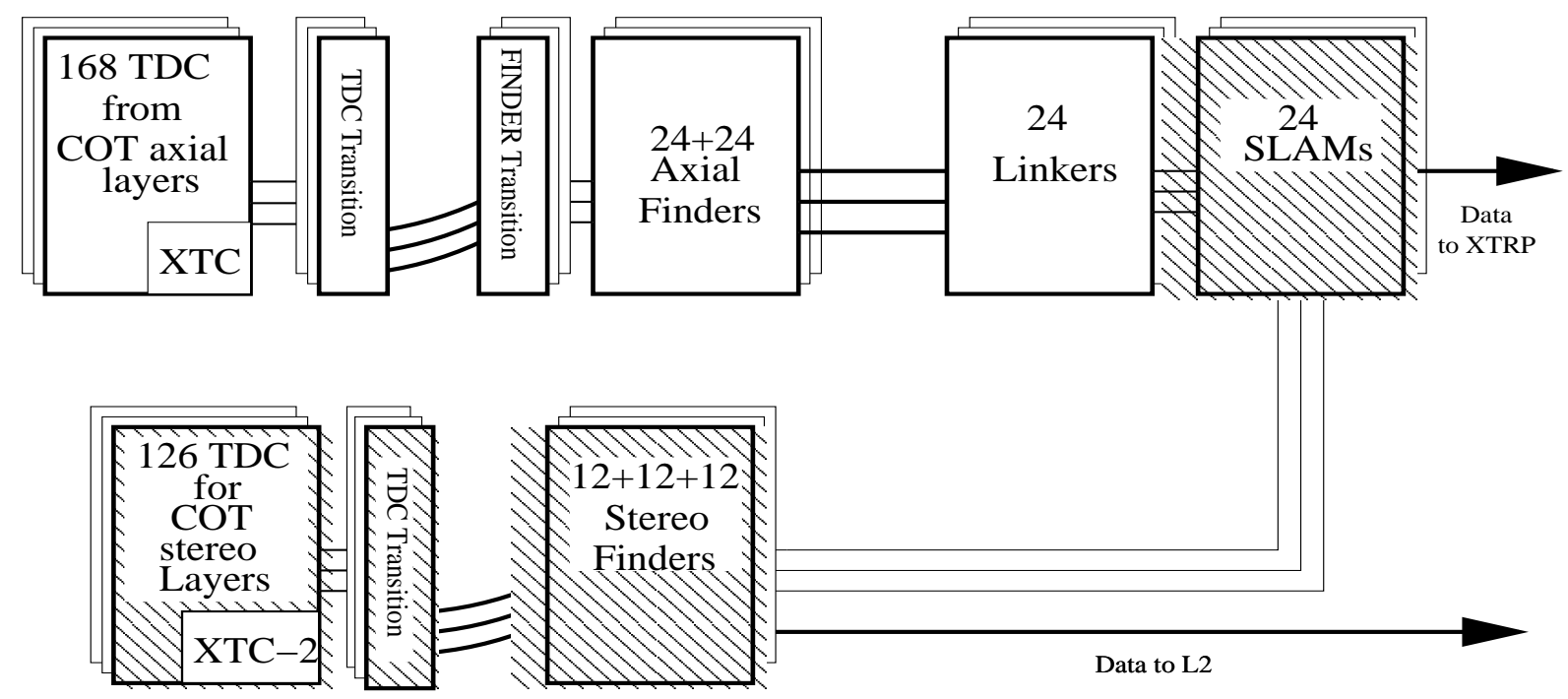

Figure 1. Schematic diagram of the XFT system architecture. The white boxes indicate the original axial system, and the shaded boxes depict the new components introduced with the upgrade. The new Stereo Linker Association Modules (SLAMs) replace the Linker Output Modules (LOMs), which are not shown.

only matched tracks will be used in the trigger selection. The more precise stereo pixels transmitted to the Level 2 trigger are matched to the axial XFT tracks, and are used to measure the track $\cot \theta$ and $z_{0}$, respectively with a resolution of $\sim 0.1$ and $10 \mathrm{~cm}$, sufficient to improve the quality of the Level 2 selection.

\section{Conclusions}

In this paper we review the upgrade of the eXtremely Fast Tracker, which reconstructs tracks in the transverse view of the CDF II Central Outer Chamber in time for the Level 1 trigger decision. The upgraded system is designed to perform a three dimensional track reconstruction. The upgraded system is in an advanced commissioning phase and it is expected to be fully operational in Summer 2006.

\section{REFERENCES}

1. E. J. Thomson et al., IEEE Trans. Nucl. Sci. 49, 1063 (2002)
2. T. Affolder et al., Nucl. Instrum. Meth. A526: 249, (2004)

3. W. Ashmanskas et al., Nucl. Instrum. Meth. A518: 532, (2004)

\section{Acknowledgments}

We would like to thank the members of the CDF II Collaboration and in particular the members of the XFT upgrade group. We thank also the organizers of $10^{\text {th }}$ Pisa Meeting on Advanced Detectors. This work has been supported by U.S. Department of Energy and National Science Foundation, Istituto Nazionale di Fisica Nucleare and by EEC RTN contract HPRN-CT-002922002. 